DECEMBER 1991, PAGES 609-625

\title{
ON UNIQUENESS FOR THE SLOW FLOW OF A DILUTE SUSPENSION OF SOLID PARTICLES IN A FLUID
}

\author{
BY \\ K. A. PERICAK-SPECTOR \\ University of Minnesota, Minneapolis, Minnesota \\ Dedicated to the memory of Jace Nunziato
}

1. Introduction. In this paper we study a set of equations which have been proposed by McTigue, Givler, and Nunziato [1] to describe the slow flow of a dilute suspension of solid particles in a fluid. We examine uniqueness in the case of an inviscid fluid (Sec. 4), a semiviscous fluid flow (Sec. 5), and a limited viscous fluid flow (Sec. 6).

2. Preliminaries.

A. Notation. We use the standard inner product $\mathbf{u} \cdot \mathbf{v}$ on $\mathbb{R}^{3} ;$ while on $\operatorname{Lin}=$ the space of all tensors, we use the inner product

$$
A \cdot B=\operatorname{tr}\left(A B^{T}\right)
$$

with $B^{T}$ the transpose of $B$ and $\operatorname{tr}$ the trace. All norms are denoted by $\|\cdot\|$ and 1 denotes the identity tensor.

The tensor product of two vectors $\mathbf{u}$ and $\mathbf{v}$, denoted by $\mathbf{u} \otimes \mathbf{v}$, is a tensor with components $(\mathbf{u} \otimes \mathbf{v})_{i j}=u_{i} v_{j}$.

We write $\nabla$ and div for the gradient and divergence operators in $\mathbb{R}^{3}$ : for a vector field $\mathbf{u}, \nabla \mathbf{u}$ is the tensor field with components $(\nabla \mathbf{u})_{i j}=\partial u_{i} / \partial x_{j}$; for a tensor field $S \operatorname{div} S$ is the vector field with components $\sum_{j=1}^{3} \partial S_{i j} / \partial x_{j}$. We write the symmetric part of $\nabla \mathbf{u}$ as $\hat{\nabla} \mathbf{u}$ :

$$
\widehat{\nabla} \mathbf{u}=\left(\nabla \mathbf{u}+(\nabla \mathbf{u})^{T}\right) / 2 .
$$

Finally, we let $\mathbf{u}_{t}$ denote the time derivative of $\mathbf{u}$.

B. The equations. We consider a dilute suspension to be a type of continuum. We assume that the particles and fluid are incompressible and we assume further that the fluid is homogeneous and isotropic. The particles are assumed to be rigid, spherical, and of uniform size with radius $a$. We also assume that the mixture is sufficiently dilute and the particles sufficiently small that Brownian motion may be important, but that collisions between particles are negligible. The balance of force and mass

Received January 18, 1989.

Author's permanent address: Department of Mathematics, Southern Illinois University, Carbondale, Illinois 62901-4408.

(C)1991 Brown University 
equations for the solid and fluid, specialized from [1], are

$$
\begin{aligned}
\gamma_{s} \phi \mathbf{u}_{t}+\gamma_{s} \phi \nabla \mathbf{u u}= & -\nabla\left(p_{s}(\phi, \psi) \phi\right)+\gamma_{s} \phi \mathbf{g}+\mathscr{F}(\phi, \psi)(\mathbf{w}-\mathbf{u}) \\
& -\mathscr{G}(\phi, \psi) \operatorname{div}(\widehat{\nabla} \mathbf{w})+p_{f}(\phi, \psi) \nabla \phi, \\
\gamma_{f} \psi \mathbf{w}_{t}+\gamma_{f} \psi \nabla \mathbf{w w}= & -\nabla\left(p_{f}(\phi, \psi) \psi\right)+\operatorname{div}(\mu(\psi) \widehat{\nabla} \mathbf{w})+\gamma_{f} \psi \mathbf{g} \\
& -\mathscr{F}(\phi, \psi)(\mathbf{w}-\mathbf{u})+\mathscr{G}(\phi, \psi) \operatorname{div}(\widehat{\nabla} \mathbf{w}) \\
& -p_{f}(\phi, \psi) \nabla \phi+\operatorname{div}(\kappa(\psi) \hat{\nabla} \mathbf{v}), \\
\phi_{t}+\operatorname{div}(\phi \mathbf{u})= & 0, \\
\psi_{t}+\operatorname{div}(\psi \mathbf{w})= & 0 .
\end{aligned}
$$

Here $\gamma_{s}$ and $\gamma_{f}$ are the actual densities of the incompressible solid and fluid, respectively, and are constant. $\phi$ and $\psi$ are the volume fractions of the solid and fluid, respectively, with $\phi, \psi>0$ and $\phi+\psi=1$. u denotes the velocity of the solid and $\mathbf{w}$ denotes the velocity of the fluid. $p_{s}$ is the hydrostatic pressure of the solid and $p_{f}$ is the hydrostatic pressure of the fluid. $\mathbf{g}$ is gravity, $\mathscr{F}$ is identified with the Stokes drag force, and $\mathscr{G}$ is associated with the Faxén force. The viscosity of the fluid is denoted by $\mu$ and $\kappa$ is an interaction viscosity. Here $\mathscr{F}, \mathscr{G}, p_{s}, p_{f}, \mu$, and $\kappa$ are positive $C^{1}$ functions of the volume fractions. Finally, $\mathbf{v}$ is the velocity of the mixture which we assume is a linear function of the sum of the solid and fluid velocities, i.e., $\mathbf{v}=\mathbf{u}+\mathbf{w}$. $^{1}$

We now suppose that the suspension occupies a region $\mathscr{B}$, with boundary $\partial \mathscr{B}$. We will let $\mathbf{n}$ denote the exterior normal to the boundary.

C. Background. The slow flow of a suspension of nearly rigid particles is seen as a natural phenomenon and in industrial processes. Previously, people involved with suspensions derived expressions for an effective viscosity [2-6] assuming that the suspension was a homogeneous mixture. Nonhomogeneous particle distribution usually exists within the flow field (cf., e.g., Parsi and Gadala-Maria [7]). The early experiments of Segré and Silberberg [8], Karnis et al. [9], and more recently the observations reported by Aoki et al. [10] illustrate particle migrations for dilute suspensions in simple flows.

It is with this end in sight, therefore, that models are being developed with which to explore the complex interactions of the fluid, particles, and boundaries of a given device. One method by which to model this phenomena is based on the continuum theory of mixtures. In the past, each constituent was assumed to be a fluid (cf., e.g., Williams [11]). More recently, classical mixture theory has been extended to situations where one or more of the constituents is disperse. Thus each "phase" is an interacting, interpenetrating continuum and hence it is possible to formulate the individual equations for conservation of mass and momentum. In addition, these conservation laws require constitutive assumptions regarding the behavior of each contributing material. Also included are interaction forces leading to lift and drag in specific flows. Finally, suitable boundary and initial conditions are found to complete

\footnotetext{
${ }^{1}$ This is not the conventional definition of $\mathbf{v}$ but is convenient for the purposes of our calculations. For further remarks see Sec. 7, or for a more detailed discussion the reader is referred to [1].
} 
the picture for the initial-boundary value problem studied in this paper.

\section{Preparing the equations.}

A. The basic problem. We will prove uniqueness for various situations of the equations of (2.1) subject to the initial and boundary conditions stated below. By a solution of (2.1) (or the appropriate modification of (2.1)) we mean functions $\mathbf{u}, \mathbf{w}: \mathscr{B} \times[0, T] \rightarrow \mathbb{R}^{3}$ and $\phi, \psi: \mathscr{B} \times[0, T] \rightarrow(0,1)$ that satisfy $(2.1)$ and the following initial and boundary conditions:

$$
\begin{aligned}
\phi(\mathbf{x}, 0) & =\phi_{0}(\mathbf{x}) \quad \text { and } \mathbf{u}(\mathbf{x}, 0)=\mathbf{u}_{0}(\mathbf{x}) \quad \text { for all } \mathbf{x} \in \mathscr{B} \\
\psi(\mathbf{x}, 0) & =\psi_{0}(\mathbf{x}) \text { and } \mathbf{w}(\mathbf{x}, 0)=\mathbf{w}_{0}(\mathbf{x}) \text { for all } \mathbf{x} \in \mathscr{B}, \\
\mathbf{u}(\mathbf{x}, t) & =\mathbf{U}(\mathbf{x}, t) \text { and } \mathbf{w}(\mathbf{x}, t)=\mathbf{W}(\mathbf{x}, t) \text { for all } \mathbf{x} \in \partial \mathscr{B} .
\end{aligned}
$$

It is unreasonable to specify the amount of fluid and particles leaving the region; that is, $\psi$ and $\phi$ cannot be specified when $\mathbf{w} \cdot \mathbf{n}>0$ and $\mathbf{u} \cdot \mathbf{n}>0$, respectively. It is, however, possible to specify the amount of fluid and particles flowing into the body. At such points $\mathbf{w} \cdot \mathbf{n}<0$ and $\mathbf{u} \cdot \mathbf{n}<0$ and here we specify that

$$
\begin{array}{ll}
\psi(\mathbf{x}, t)=\Psi(\mathbf{x}, t) & \text { for all } \mathbf{x} \in \partial \mathscr{B}_{\psi}:=\{\mathbf{x}: \mathbf{w}(\mathbf{x}, t) \cdot \mathbf{n}<0\}, \\
\phi(\mathbf{x}, t)=\Phi(\mathbf{x}, t) & \text { for all } \mathbf{x} \in \partial \mathscr{B}_{\phi}:=\{\mathbf{x}: \mathbf{u}(\mathbf{x}, t) \cdot \mathbf{n}<0\} .
\end{array}
$$

We will also note here that henceforth $p_{s}, p_{f}, \mathscr{F}, \mathscr{G}, \mu$, and $\kappa$ are assumed to be positive $C^{1}$ functions of their respective arguments.

B. Some estimates. To prove uniqueness the basic idea will be to assume that $\left(\phi_{1}, \psi_{1}, \mathbf{u}_{1}, \mathbf{w}_{1}\right)$ and $\left.\phi_{2}, \psi_{2}, \mathbf{u}_{2}, \mathbf{w}_{2}\right)$ are two solutions of (2.1) satisfying (3.1) and (3.2). Using energy estimates we will show that $\mathbf{u}_{1}(\mathbf{x}, t) \equiv \mathbf{u}_{2}(\mathbf{x}, t), \mathbf{w}_{1}(\mathbf{x}, t) \equiv$ $\mathbf{w}_{2}(x, t), \phi_{1}(\mathbf{x}, t) \equiv \phi_{2}(\mathbf{x}, t)$, and $\psi_{1}(\mathbf{x}, t) \equiv \psi_{2}(\mathbf{x}, t)$ for all $\mathbf{x} \in \mathscr{B}$ and $t \in$ $[0, T]$.

Substituting $\left(\phi_{1}, \psi_{1}, \mathbf{u}_{1}, \mathbf{w}_{1}\right)$ and $\left(\phi_{2}, \psi_{2}, \mathbf{u}_{2}, \mathbf{w}_{2}\right)$ into (2.1) and subtracting the two solid force equations, the two solid mass equations, the two fluid force equations, the two fluid mass equations, and letting $\mathbf{u}=\mathbf{u}_{1}-\mathbf{u}_{2}, \phi=\phi_{1}-\phi_{2}, \mathbf{w}=\mathbf{w}_{1}-\mathbf{w}_{2}$, $\psi=\psi_{1}-\psi_{2}, \mathbf{v}_{1}=\mathbf{u}_{1}+\mathbf{w}_{1}, \mathbf{v}_{2}=\mathbf{u}_{2}+\mathbf{w}_{2}$, and $\mathbf{v}=\mathbf{v}_{1}-\mathbf{v}_{2}$ we see, upon rearrangement of terms, that

$$
\begin{aligned}
\gamma_{s}\left(\phi_{1} \mathbf{u}_{t}\right. & \left.+\phi\left(\mathbf{u}_{2}\right)_{t}+\phi \nabla \mathbf{u}_{2} \mathbf{u}_{2}+\phi_{1} \nabla \mathbf{u} \mathbf{u}_{1}+\phi_{1} \nabla \mathbf{u}_{2} \mathbf{u}\right) \\
= & -\nabla\left(\left(p_{s 1}-p_{s 2}\right) \phi_{2}+p_{s 1} \phi\right)+\gamma_{s} \phi \mathbf{g}+\left(\mathscr{F}_{1}-\mathscr{F}_{2}\right)\left(\mathbf{w}_{2}-\mathbf{u}_{2}\right)+\mathscr{F}_{1}(\mathbf{w}-\mathbf{u}) \\
& -\mathscr{G}_{1} \operatorname{div}(\widehat{\nabla} \mathbf{w})-\left(\mathscr{G}_{1}-\mathscr{G}_{2}\right) \operatorname{div}\left(\widehat{\nabla} \mathbf{w}_{2}\right)+\left(p_{f 1}-p_{f 2}\right) \nabla \phi_{2}+p_{f 1} \nabla \phi, \\
\gamma_{f}\left(\psi_{1} \mathbf{w}_{t}\right. & \left.+\psi\left(\mathbf{w}_{2}\right)_{t}+\psi \nabla \mathbf{w}_{2} \mathbf{w}_{2}+\psi_{1} \nabla \mathbf{w} \mathbf{w}_{1}+\psi_{1} \nabla \mathbf{w}_{2} \mathbf{w}\right) \\
= & -\nabla\left(\left(p_{f 1}-p_{f 2}\right) \psi_{2}+p_{f 1} \psi\right)+\gamma_{f} \psi \mathbf{g}-\left(\mathscr{F}_{1}-\mathscr{F}_{2}\right)\left(\mathbf{w}_{2}-\mathbf{u}_{2}\right)-\mathscr{F}_{1}(\mathbf{w}-\mathbf{u}) \\
& +\mathscr{G}_{1} \operatorname{div}(\hat{\nabla} \mathbf{w})+\left(\mathscr{G}_{1}-\mathscr{G}_{2}\right) \operatorname{div}\left(\widehat{\nabla} \mathbf{w}_{2}\right)-\left(p_{f 1}-p_{f 2}\right) \nabla \phi_{2}-p_{f 1} \nabla \phi \\
& +\operatorname{div}\left[\left(\mu_{1}-\mu_{2}\right) \hat{\nabla}_{2}+\mu_{1} \hat{\nabla} \mathbf{w}+\left(\kappa_{1}-\kappa_{2}\right) \hat{\nabla}_{2}+\kappa_{1} \hat{\nabla} \mathbf{v}\right], \\
\phi_{t}=- & \operatorname{div}\left(\phi \mathbf{u}_{1}\right)-\operatorname{div}\left(\phi_{2} \mathbf{u}\right), \\
\psi_{t}=- & \operatorname{div}\left(\psi \mathbf{w}_{1}\right)-\operatorname{div}\left(\psi_{2} \mathbf{w}\right),
\end{aligned}
$$

where $p_{s i}=p_{s}\left(\phi_{i}, \psi_{i}\right)$ (or $p_{s}\left(\phi_{i}\right)$ where appropriate), $p_{f i}=p_{f}\left(\phi_{i}, \psi_{i}\right)$ (or $p_{f}\left(\psi_{i}\right)$ 
where appropriate), $\mathscr{F}_{i}=\mathscr{F}\left(\phi_{i}, \psi_{i}\right), \mathscr{G}_{i}=\mathscr{G}\left(\phi_{i}, \psi_{i}\right), \mu_{i}=\mu\left(\psi_{i}\right)$, and $\kappa_{i}=\kappa\left(\psi_{i}\right)$ for $i=1,2$.

Notice that $\phi, \psi, \mathbf{u}$, and $\mathbf{w}$ satisfy the following initial and boundary conditions:

$$
\begin{array}{rlrl}
\phi(\mathbf{x}, 0) & =0 \quad \text { and } & \mathbf{u}(\mathbf{x}, 0)=\mathbf{0} & \text { for all } \mathbf{x} \in \mathscr{B}, \\
\psi(\mathbf{x}, 0) & =0 \quad \text { and } & \mathbf{w}(\mathbf{x}, 0)=\mathbf{0} \text { for all } \mathbf{x} \in \mathscr{B}, \\
\mathbf{u}(\mathbf{x}, t) & =\mathbf{w}(\mathbf{x}, t)=\mathbf{0} & & \text { for all } \mathbf{x} \in \partial \mathscr{B}, \\
\phi(\mathbf{x}, t)=0 & & \text { for all } \mathbf{x} \in \partial \mathscr{B}_{\phi}, \\
\psi(\mathbf{x}, t)=0 & & \text { for all } \mathbf{x} \in \partial \mathscr{B}_{\psi} .
\end{array}
$$

Multiplying $(3.3)_{3}$ by $\phi_{1} \phi$, integrating over $\mathscr{B}$, rewriting the right-hand side, and applying the divergence theorem to the appropriate terms we obtain

$$
\begin{aligned}
\frac{d}{d t} \int_{\mathscr{B}} \phi_{1} \phi^{2} / 2= & -\int_{\mathscr{B}} \phi_{1} \phi^{2} \operatorname{div} \mathbf{u}_{1}-\int_{\mathscr{B}} \phi_{1} \phi\left(\nabla \phi_{2}\right) \cdot \mathbf{u} \\
& -\int_{\mathscr{B}} \phi_{1} \phi_{2} \phi \operatorname{div} \mathbf{u}+N P_{1},
\end{aligned}
$$

where $N P_{1}=-\int_{\mathscr{B}} \phi^{2} \phi_{1} \mathbf{u}_{1} \cdot \mathbf{n} / 2$. From before we see that $\phi_{i}$ cannot be specified where $\mathbf{u}_{i} \cdot \mathbf{n}>0$. However at such points $N P_{1}<0$. Recall also that $\phi=0$ on $\partial \mathscr{B}_{\phi}$, whence at such points $N P_{1}=0$. Thus $N P_{1}$ is nonpositive.

Throughout this paper we will need to bound integrals with terms involving $\phi^{2}$, $\|\mathbf{u}\|^{2}, \psi^{2}$, and $\|\mathbf{w}\|^{2}$ and we will frequently use the following arithmetic-geometric mean inequality:

$$
\mathbf{y} \cdot \mathbf{z} \leq \frac{\varepsilon}{2}\|\mathbf{y}\|^{2}+\frac{1}{2 \varepsilon}\|\mathbf{z}\|^{2}
$$

where $\varepsilon>0$.

Applying (3.6) to the second and third integrals (on the right-hand side) of (3.5) and noting that $(\operatorname{div} \mathbf{f})^{2} \leq 9\|\hat{\nabla} \mathbf{f}\|^{2}$ we see that

$$
\frac{d}{d t} \int_{\mathscr{B}} \phi_{1} \phi^{2} / 2 \leq \int_{\mathscr{B}}\left[c_{1} \phi^{2}+\|\mathbf{u}\|^{2}+9 \varepsilon_{1}\|\widehat{\nabla} \mathbf{u}\|^{2}\right] / 2,
$$

where $c_{1}=\left(-2 \phi_{1} \operatorname{div} \mathbf{u}_{1}+\left\|\phi_{1} \nabla \phi_{2}\right\|^{2}+\left(\phi_{1} \phi_{2}\right)^{2} / \varepsilon_{1}\right)$ and $\varepsilon_{1}$ is an arbitrary constant to be determined later.

REMARK 3.1. Henceforth $\varepsilon_{i}, i=1,2, \ldots$, will be an arbitrary constant to be determined later.

Similarly, multiplying $(3.3)_{4}$ by $\psi_{1} \psi$, integrating over $\mathscr{B}$, applying the divergence theorem, and bounding the appropriate terms we arrive at

$$
\frac{d}{d t} \int_{\mathscr{B}} \psi_{1} \psi^{2} / 2 \leq \int_{\mathscr{B}}\left[c_{2} \psi^{2}+\|\mathbf{w}\|^{2}+9 \varepsilon_{2}\|\hat{\nabla} \mathbf{w}\|^{2}\right] / 2
$$

where $c_{2}=\left(-2 \psi_{1} \operatorname{div} \mathbf{w}_{1}+\left\|\psi_{1} \nabla \psi_{2}\right\|^{2}+\left(\psi_{1} \psi_{2}\right)^{2} / \varepsilon_{2}\right)$.

Let us now turn our attention to (3.3) 1,2 . Taking the inner product of $(3.3)_{2}$ with w, integrating over $\mathscr{B}$, applying the divergence theorem, and rearranging terms we 
obtain

$$
\begin{aligned}
\frac{d}{d t} \int_{\mathscr{B}} \gamma_{f} \psi_{1}\|\mathbf{w}\|^{2} / 2= & \int_{\mathscr{B}} \psi \mathbf{a} \cdot \mathbf{w}-\int_{\mathscr{B}} \gamma_{f} \psi_{1} \nabla \mathbf{w}_{2} \mathbf{w} \cdot \mathbf{w} \\
& -\int_{\mathscr{B}} \mathscr{F}_{1}(\mathbf{w}-\mathbf{u}) \cdot \mathbf{w}-\int_{\mathscr{B}}\left(\mathscr{F}_{1}-\mathscr{F}_{2}\right)\left(\mathbf{w}_{2}-\mathbf{u}_{2}\right) \cdot \mathbf{w} \\
& +\int_{\mathscr{B}}\left(\mathscr{G}_{1}-\mathscr{G}_{2}\right)\left(\operatorname{div} \hat{\nabla} \mathbf{w}_{2}\right) \cdot \mathbf{w} \\
& -\int_{\mathscr{B}}\left[\left(\mu_{1}-\mu_{2}\right) \hat{\nabla}_{\mathbf{w}}+\left(\kappa_{1}-\kappa_{2}\right) \hat{\nabla} \mathbf{v}_{2}\right] \cdot \hat{\nabla} \mathbf{w} \\
& -\int_{\mathscr{B}} \hat{\nabla} \mathbf{w} \cdot \mathbf{w} \otimes \nabla \mathscr{G}_{1}-\int_{\mathscr{B}}\left(\mathscr{G}_{1}+\mu_{1}\right)\|\hat{\nabla} \mathbf{w}\|^{2}-\int_{\mathscr{B}} \kappa_{1} \hat{\nabla} \mathbf{v} \cdot \hat{\nabla} \mathbf{w} \\
& +\int_{\mathscr{B}}\left(p_{f 1}-p_{f 2}\right)\left(\nabla \psi_{2}\right) \cdot \mathbf{w}+\int_{\mathscr{B}}\left(p_{f 1}-p_{f 2}\right) \psi_{2} \operatorname{div} \mathbf{w},
\end{aligned}
$$

where $\mathbf{a}=\left[\gamma_{f}\left(\mathbf{g}-\left(\mathbf{w}_{2}\right)_{t}-\nabla \mathbf{w}_{2} \mathbf{w}_{2}\right)-\nabla p_{f 1}\right]$. Here we have used the fact that $\phi_{i}+\psi_{i}=1$ and hence that $p_{f 1} \nabla\left(\phi_{i}+\psi_{i}\right)=0$.

We will now bound the integrals on the right-hand side of (3.9). Applying (3.6), the first integral is bounded by

$$
\int_{\mathscr{B}}\left(c_{3} \psi^{2}+\|\mathbf{w}\|^{2}\right) / 2,
$$

where $c_{3}=\|\mathbf{a}\|^{2}$. The second and third integrals are bounded by

$$
\int_{\mathscr{B}} \gamma_{f} \psi_{1}\left|\lambda_{m w}\right|\|\mathbf{w}\|^{2},
$$

and

$$
\int_{\mathscr{B}} \mathscr{F}_{1}\left(\|\mathbf{u}\|^{2}-\|\mathbf{w}\|^{2}\right) / 2,
$$

respectively, where $\lambda_{m w}$ is the minimum eigenvalue of $\hat{\nabla} \mathbf{w}_{2}$.

Recall that $\mathscr{F}, \mathscr{G}, \mu, \kappa \in C^{1}$ and so by applying the Mean Value Theorem we have that

$$
\begin{aligned}
& \mathscr{F}_{1}-\mathscr{F}_{2}:=\mathscr{F}\left(\phi_{1}, \psi_{1}\right)-\mathscr{F}\left(\phi_{2}, \psi_{2}\right)=\mathscr{F}_{\phi}\left(\xi_{1}, \xi_{2}\right) \phi+\mathscr{F}_{\psi}\left(\xi_{1}, \xi_{2}\right) \psi, \\
& \mathscr{G}_{1}-\mathscr{G}_{2}:=\mathscr{G}\left(\phi_{1}, \psi_{1}\right)-\mathscr{G}\left(\phi_{2}, \psi_{2}\right)=\mathscr{G}_{\phi}\left(\xi_{3}, \xi_{4}\right) \phi+\mathscr{G}_{\psi}\left(\xi_{3}, \xi_{4}\right) \psi, \\
& \mu_{1}-\mu_{2}:=\mu\left(\psi_{1}\right)-\mu\left(\psi_{2}\right)=\mu^{\prime}\left(\xi_{5}\right) \psi, \\
& \kappa_{1}-\kappa_{2}:=\kappa\left(\psi_{1}\right)-\kappa\left(\psi_{2}\right)=\kappa^{\prime}\left(\xi_{6}\right) \psi,
\end{aligned}
$$

where $\mathscr{F}_{\phi}=\partial \mathscr{F} / \partial \phi, \mathscr{F}_{\psi}=\partial \mathscr{F} / \partial \psi, \mathscr{G}_{\phi}=\partial \mathscr{G} / \partial \phi, \mathscr{G}_{\psi}=\partial \mathscr{G} / \partial \psi, \mu^{\prime}=d \mu / \partial \psi$, $\kappa^{\prime}=d \kappa / d \psi$, and $\xi_{i} \in(0,1), i=1, \ldots, 6$. Hence the fourth, fifth, and sixth integrals are bounded from above by

$$
\begin{aligned}
& \int_{\mathscr{B}}\left[c_{4} \phi^{2}+c_{5} \psi^{2}+2\|\mathbf{w}\|^{2}\right] / 2, \\
& \int_{\mathscr{B}}\left[c_{6} \phi^{2}+c_{7} \psi^{2}+2\|\mathbf{w}\|^{2}\right] / 2, \\
& \int_{\mathscr{B}}\left[c_{8} \psi^{2}+\varepsilon_{3}\|\widehat{\nabla} \mathbf{w}\|^{2}\right] / 2,
\end{aligned}
$$


respectively, where $c_{4}=\left\|\mathscr{F}_{\phi}\left(\xi_{1}, \xi_{2}\right)\left(\mathbf{w}_{2}-\mathbf{u}_{2}\right)\right\|^{2}, c_{5}=\left\|\mathscr{F}_{\psi}\left(\xi_{1}, \xi_{2}\right)\left(\mathbf{w}_{2}-\mathbf{u}_{2}\right)\right\|^{2}, c_{6}=$ $\left\|\mathscr{G}_{\phi}\left(\xi_{3}, \xi_{4}\right) \operatorname{div} \hat{\nabla} \mathbf{w}_{2}\right\|^{2}, c_{7}=\left\|\mathscr{G}_{\psi}\left(\xi_{3}, \xi_{4}\right) \operatorname{div} \hat{\nabla} \mathbf{w}_{2}\right\|^{2}$, and $c_{8}=\left\|\mu^{\prime}\left(\xi_{5}\right) \hat{\nabla} \mathbf{w}_{2}+\kappa^{\prime}\left(\xi_{6}\right) \hat{\nabla} \mathbf{v}_{2}\right\|^{2}$ $/ \varepsilon_{3}$.

Finally the seventh integral is bounded by

$$
\int_{\mathscr{B}}\left[c_{9}\|\mathbf{w}\|^{2}+\varepsilon_{4}\|\widehat{\nabla} \mathbf{w}\|^{2}\right] / 2
$$

where $c_{9}=\left\|\nabla \mathscr{G}_{1}\right\|^{2} / \varepsilon_{4}$. Using (3.10)-(3.12), and (3.14)-(3.17) we have that

$$
\begin{aligned}
\frac{d}{d t} \int_{\mathscr{B}} \gamma_{f} \psi_{1}\|\mathbf{w}\|^{2} / 2 \leq & \int_{\mathscr{B}}\left[c_{3}+c_{5}+c_{7}+c_{8}\right] \psi^{2} / 2+\int_{\mathscr{B}}\left[c_{4}+c_{6}\right] \phi^{2} / 2 \\
& +\int_{\mathscr{B}}\left[5+2 \gamma_{f} \psi_{1}\left|\lambda_{m w}\right|-\mathscr{F}_{1}+c_{9}\right]\|\mathbf{w}\|^{2} / 2 \\
& +\int_{\mathscr{B}} \mathscr{F}_{1}\|\mathbf{u}\|^{2} / 2+\int_{\mathscr{B}}\left[\varepsilon_{3}+\varepsilon_{4}-2\left(\mathscr{G}_{1}+\mu_{1}\right)\right]\|\hat{\nabla} \mathbf{w}\|^{2} / 2 \\
& -\int_{\mathscr{B}} \kappa_{1} \hat{\nabla} \mathbf{v} \cdot \hat{\nabla} \mathbf{w}+\int_{\mathscr{B}}\left(p_{f 1}-p_{f 2}\right) \nabla \psi_{2} \cdot \mathbf{w} \\
& +\int_{\mathscr{B}}\left(p_{f 1}-p_{f 2}\right) \psi_{2} \operatorname{div} \mathbf{w} .
\end{aligned}
$$

If we now take the inner product of $(3.3)_{1}$ with $\mathbf{u}$, integrate over $\mathscr{B}$, and apply the above ideas to the resulting equation we arrive at

$$
\begin{aligned}
\frac{d}{d t} \int_{\mathscr{B}} \gamma_{s} \phi_{1}\|\mathbf{u}\|^{2} / 2= & \int_{\mathscr{B}} \phi \mathbf{b} \cdot \mathbf{u}-\int_{\mathscr{B}} \gamma_{s} \phi_{1} \nabla \mathbf{u}_{2} \mathbf{u} \cdot \mathbf{u} \\
& +\int_{\mathscr{B}} \mathscr{F}_{1}(\mathbf{w}-\mathbf{u}) \cdot \mathbf{u}+\int_{\mathscr{B}}\left(\mathscr{F}_{1}-\mathscr{F}_{2}\right)\left(\mathbf{w}_{2}-\mathbf{u}_{2}\right) \cdot \mathbf{u} \\
& -\int_{\mathscr{B}}\left(\mathscr{G}_{1}-\mathscr{G}_{2}\right) \operatorname{div} \hat{\nabla} \mathbf{w}_{2} \cdot \mathbf{u}+\int_{\mathscr{B}} \hat{\nabla} \mathbf{w} \cdot \mathbf{u} \otimes \nabla \mathscr{G}_{1} \\
& +\int_{\mathscr{B}} \mathscr{G}_{1} \hat{\nabla} \mathbf{w} \cdot \hat{\nabla} \mathbf{u}+\int_{\mathscr{B}}\left(p_{f 1}-p_{f 2}\right) \nabla \phi_{2} \cdot \mathbf{u} \\
& +\int_{\mathscr{B}}\left[\left(p_{s 1}-p_{s 2}\right) \phi_{2}+\left(p_{s 1}-p_{f 1}\right) \phi\right] \operatorname{div} \mathbf{u},
\end{aligned}
$$

or

$$
\begin{aligned}
\frac{d}{d t} \int_{\mathscr{B}} \gamma_{s} \phi_{1}\|\mathbf{u}\|^{2} / 2 \leq & \int_{\mathscr{B}}\left[c_{4}+c_{6}+c_{10}\right] \phi^{2} / 2+\int_{\mathscr{B}}\left[c_{5}+c_{7}\right] \psi^{2} / 2 \\
& +\int_{\mathscr{B}}\left[5+2 \gamma_{s} \phi_{1}\left|\lambda_{m u}\right|+c_{11}-\mathscr{F}_{1}\right]\|\mathbf{u}\|^{2} / 2 \\
& +\int_{\mathscr{B}} \mathscr{F}_{1}\|\mathbf{w}\|^{2} / 2+\int_{\mathscr{B}} \varepsilon_{5}\|\hat{\nabla} \mathbf{w}\|^{2} / 2+\int_{\mathscr{B}} \mathscr{G}_{1} \hat{\nabla} \mathbf{w} \cdot \hat{\nabla} \mathbf{u} \\
& +\int_{\mathscr{B}}\left(p_{f 1}-p_{f 2}\right) \nabla \phi_{2} \cdot \mathbf{u} \\
& +\int_{\mathscr{B}}\left[\left(p_{s 1}-p_{s 2}\right) \phi_{2}+\left(p_{s 1}-p_{f 1}\right) \phi\right] \operatorname{div} \mathbf{u},
\end{aligned}
$$

where $\mathbf{b}=\left[\gamma_{s}\left(\mathbf{g}-\left(\mathbf{u}_{2}\right)_{t}-\nabla \mathbf{u}_{2} \mathbf{u}_{2}\right)-\nabla p_{f 1}\right], c_{10}=\|\mathbf{b}\|^{2}, c_{11}=\left\|\nabla \mathscr{G}_{1}\right\|^{2} / \varepsilon_{5}$, and $\lambda_{m u}$ is he minimum eigenvalue of $\hat{\nabla} \mathbf{u}_{2}$. 
Depending on the various circumstances we will use slight modifications of (3.18) and (3.19) to prove uniqueness for an inviscid fluid flow, a semi-viscous fluid flow, and a limited viscosity fluid flow.

4. Inviscid fluid flow. We will now consider the problem of uniqueness for an inviscid fluid flow. Here $\mathscr{G} \equiv \mu \equiv \kappa \equiv 0$. We will further assume that $p_{s}$ and $p_{f}$ are strictly monotonically increasing with $p_{s}=p_{s}(\phi)>p_{f}(\psi)=p_{f} \quad\left(=p_{f}(1-\phi)\right)$. It is now possible to state and prove the following uniqueness theorem.

TheOREM 1. For every $T>0$ there is at most one classical solution of

$$
\begin{aligned}
\gamma_{s} \phi \mathbf{u}_{t}+\gamma_{s} \phi \nabla \mathbf{u u} & =-\nabla\left(p_{s} \phi\right)+\gamma_{s} \phi \mathbf{g}+\mathscr{F}(\mathbf{w}-\mathbf{u})+p_{f} \nabla \phi, \\
\gamma_{f} \psi \mathbf{w}_{t}+\gamma_{f} \psi \nabla \mathbf{w w} & =-\psi \nabla p_{f}+\gamma_{f} \psi \mathbf{g}-\mathscr{F}(\mathbf{w}-\mathbf{u}), \\
\phi_{t}+\operatorname{div}(\phi \mathbf{u}) & =0, \quad \psi_{t}+\operatorname{div}(\psi \mathbf{w})=0,
\end{aligned}
$$

satisfying (3.1) and (3.2) on $[0, T]$.

Proof. Consider (3.18) and (3.19). Since $\mathscr{G} \equiv \mu \equiv \kappa \equiv 0$ we need only set $c_{6}=c_{7}=c_{8}=c_{9}=c_{11}=\varepsilon_{3}=\varepsilon_{4}=\varepsilon_{5}=\mathscr{G}_{1}=\mu_{1}=\kappa_{1}=0$. Adding the resulting equations together we arrive at

$$
\begin{aligned}
\frac{d}{d t} \int_{\mathscr{B}}\left[\gamma_{f} \psi_{1}\|\mathbf{w}\|^{2}+\gamma_{s} \phi_{1}\|\mathbf{u}\|^{2}\right] / 2 & \left.+\left[5+2 \gamma_{s} \phi_{1}\left|\lambda_{m u}\right|\right]\|\mathbf{u}\|^{2}\right\} / 2 \\
\leq & \int_{\mathscr{B}}\left\{\left[c_{3}+2 c_{5}\right] \psi^{2}+\left[2 c_{4}+c_{10}\right] \phi^{2}+\left[5+2 \gamma_{f} \psi_{1}\left|\lambda_{m w}\right|\right]\|\mathbf{w}\|^{2}\right. \\
& +\int_{\mathscr{B}}\left(p_{f 1}-p_{f 2}\right)\left(\nabla \psi_{2} \cdot \mathbf{w}+\nabla \phi_{2} \cdot \mathbf{u}\right) \\
& +\int_{\mathscr{B}}\left(p_{f 1}-p_{f 2}\right) \psi_{2} \operatorname{div} \mathbf{w} \\
& +\int_{\mathscr{B}}\left(\left(p_{s 1}-p_{s 2}\right) \phi_{2}+\left(p_{s 1}-p_{f 1}\right) \phi\right) \operatorname{div} \mathbf{u} .
\end{aligned}
$$

REMARK 4.1. It is actually possible to get a better bound on $\|\mathbf{w}\|^{2} / 2$. Since $\mathscr{G} \equiv 0$, we no longer need (3.15). Hence the coefficient is $\left(3+2 \gamma_{f} \psi_{1}\left|\lambda_{m w}\right|\right)$. Correspondingly the coefficient of $\|\mathbf{u}\|^{2} / 2$ is $\left(3+2 \gamma_{s} \phi_{1}\left|\lambda_{m u}\right|\right)$. The stricter bound is not necessary for our computations.

We now need to bound the last three integrals. Using the Mean Value Theorem we see that

$$
\begin{gathered}
p_{s 1}-p_{s 2}:=p_{s}\left(\phi_{1}\right)-p_{s}\left(\phi_{2}\right)=p_{s}^{\prime}\left(\xi_{7}\right) \phi, \\
p_{f 1}-p_{f 2}:=p_{f}\left(\psi_{1}\right)-p_{f}\left(\psi_{2}\right)=p_{f}^{\prime}\left(\xi_{8}\right) \psi,
\end{gathered}
$$

where $p_{s}^{\prime}=d p_{s} / d \phi, p_{f}^{\prime}=d p_{f} / d \psi$, and $\xi_{7}, \xi_{8} \in(0,1)$. Thus

$$
\int_{\mathscr{B}}\left(p_{f 1}-p_{f 2}\right)\left(\nabla \psi_{2} \cdot \mathbf{w}+\nabla \phi_{2} \cdot \mathbf{u}\right) \leq \int_{\mathscr{B}} c_{12} \psi^{2}+\int_{\mathscr{B}}\left[\|\mathbf{u}\|^{2}+\|\mathbf{w}\|^{2}\right] / 2,
$$

where $c_{12}=\left\|p_{f}^{\prime}\left(\xi_{8}\right) \nabla \psi_{2}\right\|^{2}$ and we have used the fact that $\nabla \phi_{2}=-\nabla \psi_{2}$. 
Let us now rewrite the last two integrals as

$$
\int_{\mathscr{B}} \beta_{2} \psi \operatorname{div} \mathbf{w}+\int_{\mathscr{B}} \beta_{1} \phi \operatorname{div} \mathbf{u},
$$

where $\beta_{1}=\left(p_{s}^{\prime}\left(\xi_{7}\right) \phi_{2}+p_{s 1}-p_{f 1}\right)$ and $\beta_{2}=p_{f}^{\prime}\left(\xi_{8}\right) \psi_{2}$. Recall that $p_{s}$ and $p_{f}$ are strictly monotonically increasing and that $p_{s 1}-p_{f 1}>0$, hence $\beta_{1}$ and $\beta_{2}$ are strictly positive. Consider now the following difference of two balance of mass equations:

$$
\alpha_{t}+(\nabla \alpha) \cdot \mathbf{V}_{1}+\alpha \operatorname{div} \mathbf{V}_{1}+\left(\nabla \alpha_{2}\right) \cdot \mathbf{V}+\alpha_{2} \operatorname{div} \mathbf{V}=0,
$$

where $(\alpha, \mathbf{V})=(\phi, \mathbf{u})$ in $(3.3)_{3}$ and $(\alpha, \mathbf{V})=(\psi, \mathbf{w})$ in (3.3 $)_{4}$. Multiply this equation by $\alpha$ and rearrange terms to obtain

$$
\alpha \operatorname{div} \mathbf{V}=-\frac{1}{\alpha_{2}}\left(\left[\frac{\alpha^{2}}{2}\right]_{V_{1}}^{\circ}+\left(\alpha \nabla \alpha_{2}\right) \cdot \mathbf{V}+\alpha^{2} \operatorname{div} \mathbf{V}_{1}\right)
$$

where []$_{V_{1}}^{\circ}$ denotes the material derivative with respect to $\mathbf{V}_{1}$ (i.e., $[f]_{V_{1}}^{\circ}=f_{t}+$ $\left.(\nabla f) \cdot \mathbf{V}_{1}\right)$. Then for some $\beta$ we have that

$$
\int_{\mathscr{B}} \beta \alpha \operatorname{div} \mathbf{V}=-\int_{\mathscr{B}} \frac{\beta}{\alpha_{2}}\left[\frac{\alpha^{2}}{2}\right]_{V_{1}}^{\circ}-\int_{\mathscr{B}}\left(\frac{\beta}{\alpha_{2}} \nabla \alpha_{2}\right) \cdot \alpha \mathbf{V}-\int_{\mathscr{B}} \frac{\beta}{\alpha_{2}} \alpha^{2} \operatorname{div} \mathbf{V}_{1} .
$$

Following Graffi [12] we see that if $f_{1}=\beta_{1} \phi^{2} / 2 \phi_{1} \phi_{2}$, then we have that

$$
\begin{aligned}
\frac{d}{d t} \int_{\mathscr{B}} \phi_{1} f_{1} & =-\int_{\partial \mathscr{B}} f_{1} \phi_{1} \mathbf{u}_{1} \cdot \mathbf{n}+\int_{\mathscr{B}} \phi_{1}\left[f_{1}\right]_{u_{1}}^{\circ} \\
& =-\int_{\partial \mathscr{B}} \frac{\beta_{1}}{\phi_{2}} \frac{\phi^{2}}{2} \mathbf{u}_{1} \cdot \mathbf{n}+\int_{\mathscr{B}} \phi_{1}\left(\left[\frac{\beta_{1}}{\phi_{1} \phi_{2}}\right]_{u_{1}}^{\circ} \frac{\phi^{2}}{2}+\frac{\beta_{1}}{\phi_{1} \phi_{2}}\left[\frac{\phi^{2}}{2}\right]_{u_{1}}^{\circ}\right) .
\end{aligned}
$$

Thus

$$
\begin{aligned}
\int_{\mathscr{B}} \beta_{1} \phi \operatorname{div} \mathbf{u}= & -\int_{\mathscr{B}} \phi\left(\frac{\beta_{1}}{\phi_{2}} \nabla \phi_{2}\right) \cdot \mathbf{u} \\
& +\int_{\mathscr{B}}\left(\phi_{1}\left[\frac{\beta_{1}}{\phi_{1} \phi_{2}}\right]_{u_{1}}^{\circ}-2 \frac{\beta_{1}}{\phi_{2}} \operatorname{div} \mathbf{u}_{1}\right) \frac{\phi^{2}}{2}-\frac{d}{d t} \int_{\mathscr{B}} \frac{\beta_{1}}{\phi_{2}} \frac{\phi^{2}}{2}+N P_{2},
\end{aligned}
$$

where $N P_{2}=-\int_{\partial \mathscr{B}} \beta_{1} \phi^{2} \mathbf{u}_{1} \cdot \mathbf{n} / 2 \phi_{2}$. From before (see Sec. 3) we see that $N P_{2}$ is nonpositive. Using (3.6) on the first integral we arrive at

$$
\int_{\mathscr{B}} \beta_{1} \phi \operatorname{div} \mathbf{u} \leq \int_{\mathscr{B}} c_{13} \phi^{2} / 2+\int_{\mathscr{B}}\|\mathbf{u}\|^{2} / 2-\frac{d}{d t} \int_{\mathscr{B}}\left(\frac{\beta_{1}}{\phi_{2}} \frac{\phi^{2}}{2}\right),
$$

where $c_{13}=\left(\left\|\left(\beta_{1} \nabla \phi_{2}\right) / \phi_{2}\right\|^{2}+\phi_{1}\left[\beta_{1} / \phi_{1} \phi_{2}\right]_{u_{1}}^{0}-2\left(\beta_{1} \operatorname{div} \mathbf{u}_{1}\right) / \phi_{2}\right)$. Similarly,

$$
\int_{\mathscr{B}} \beta_{2} \psi \operatorname{div} \mathbf{w} \leq \int_{\mathscr{B}} c_{14} \psi^{2} / 2+\int_{\mathscr{B}}\|\mathbf{w}\|^{2} / 2-\frac{d}{d t} \int_{\mathscr{B}}\left(\frac{\beta_{2}}{\psi_{2}} \frac{\psi^{2}}{2}\right),
$$

where $c_{14}=\left(\left\|\left(\beta_{2} \nabla \psi_{2}\right) / \psi_{2}\right\|^{2}+\psi_{1}\left[\beta_{2} / \psi_{2} \psi_{2}\right]_{w_{1}}^{\circ}-2\left(\beta_{2} \operatorname{div} \mathbf{w}_{1}\right) / \psi_{2}\right)$. 
Thus, using (4.3)-(4.5), Eq. (4.1) becomes

$$
\begin{aligned}
& \frac{d}{d t} \int_{\mathscr{B}}\left[\gamma_{s} \phi_{1}\|\mathbf{u}\|^{2}+\gamma_{f} \psi_{1}\|\mathbf{w}\|^{2}+\beta_{1} \phi^{2} / \phi_{2}+\beta_{2} \psi^{2} / \psi_{2}\right] \\
& \quad \leq \int_{\mathscr{B}}\left[K_{1} \gamma_{s} \phi_{1}\|\mathbf{u}\|^{2}+K_{2} \gamma_{f} \psi_{1}\|\mathbf{w}\|^{2}+K_{3} \beta_{1} \phi^{2} / \phi_{2}+K_{4} \beta_{2} \psi^{2} / \psi_{2}\right],
\end{aligned}
$$

where

$$
\begin{aligned}
& K_{1}=\sup _{\mathbf{x} \in \mathscr{B}}\left\{\left(7+2 \gamma_{s} \phi_{1}\left|\lambda_{m u}\right|\right) / \gamma_{s} \phi_{1}\right\}, \\
& K_{2}=\sup _{\mathbf{x} \in \mathscr{B}}\left\{\left(7+2 \gamma_{f} \psi_{1}\left|\lambda_{m w}\right|\right) / \gamma_{f} \psi_{1}\right\}, \\
& K_{3}=\sup _{\mathbf{x} \in \mathscr{B}}\left\{\left(2 c_{4}+c_{10}+c_{13}\right) \phi_{2} / \beta_{1}\right\}, \\
& K_{4}=\sup _{\mathbf{x} \in \mathscr{B}}\left\{\left(c_{3}+2 c_{5}+c_{12}+c_{14}\right) \psi_{2} / \beta_{2}\right\} .
\end{aligned}
$$

Setting $E_{1}(t):=\int_{\mathscr{B}}\left[\gamma_{s} \phi_{1}\|\mathbf{u}\|^{2}+\gamma_{f} \psi_{1}\|\mathbf{w}\|^{2}+\beta_{1} \phi^{2} / \phi_{2}+\beta_{2} \psi^{2} / \psi_{2}\right]$ and $M_{1}=$ $\sup _{t \in[0, T]}\left\{K_{1}, K_{2}, K_{3}, K_{4}\right\}$ we obtain $d E_{1} / d t \leq M_{1} E_{1}$ so that

$$
E_{1}(t) \leq E(0) e^{M_{1} t} \equiv 0
$$

Therefore $E_{1}(t) \equiv 0$ for all $t \in[0, T]$, and $\mathbf{u}(\mathbf{x}, t)=\mathbf{w}(\mathbf{x}, t)=\mathbf{0}$ and $\phi(\mathbf{x}, t)=$ $\psi(\mathbf{x}, t)=0$. Whence $\mathbf{u}_{1}(\mathbf{x}, t)=\mathbf{u}_{2}(\mathbf{x}, t), \mathbf{w}_{1}(\mathbf{x}, t)=\mathbf{w}_{2}(\mathbf{x}, t), \phi_{1}(\mathbf{x}, t)=\phi_{2}(\mathbf{x}, t)$, and $\psi_{1}(\mathbf{x}, t)=\psi_{2}(\mathbf{x}, t)$ for all $\mathbf{x} \in \mathscr{B}$ and $t \in[0, T]$.

5. Semi-viscous fluid flow. Let us consider the problem of uniqueness for a semiviscous fluid flow. Recall that in this type of flow there is no viscosity effect from the solid. Hence there is no viscosity term in the solid equation $(\mathscr{G} \equiv 0)$ and the mixture velocity, $\mathbf{v}$, depends only on the velocity of the fluid, $\mathbf{w}$. We shall assume further that $p_{s}$ is a strictly monotonically increasing function of $\phi, p_{f}=p_{f}(\phi, \psi)$ with $p_{s}>p_{f}$. We can now state and prove the following

THEOREM 2. For every $T>0$ there is at most one classical solution of

$$
\begin{aligned}
\gamma_{s} \phi \mathbf{u}_{t}+\gamma_{s} \phi \nabla \mathbf{u u} & =-\nabla\left(p_{s} \phi\right)+\gamma_{s} \phi \mathbf{g}+\mathscr{F}(\mathbf{w}-\mathbf{u})+p_{f} \nabla \phi, \\
\gamma_{f} \psi \mathbf{w}_{t}+\gamma_{f} \psi \nabla \mathbf{w w} & =-\psi \nabla p_{f}+\gamma_{f} \psi \mathbf{g}-\mathscr{F}(\mathbf{w}-\mathbf{u})+\operatorname{div}((\mu+\kappa) \hat{\nabla} \mathbf{w}), \\
\phi_{t}+\operatorname{div}(\phi \mathbf{u}) & =0, \quad \psi_{t}+\operatorname{div}(\psi \mathbf{w})=0,
\end{aligned}
$$

satisfying (3.1) and (3.2) on $[0, T]$. 
Proof. Consider (3.8), (3.18), and (3.19). Since $\mathscr{G} \equiv 0$ we set $c_{6}=c_{7}=c_{9}=$ $c_{11}=\mathscr{G}_{1}=\varepsilon_{4}=\varepsilon_{5}=0$. Adding the resulting equations together we arrive at

$$
\begin{aligned}
\frac{d}{d t} \int_{\mathscr{B}} & \left(\psi_{1} \psi^{2}+\gamma_{f} \psi_{1}\|\mathbf{w}\|^{2}+\gamma_{s} \phi_{1}\|\mathbf{u}\|^{2}\right) / 2 \\
\leq & \int_{\mathscr{B}}\left(c_{2}+c_{3}+2 c_{5}+c_{8}\right) \psi^{2} / 2+\int_{\mathscr{B}}\left(2 c_{4}+c_{10}\right) \phi^{2} / 2 \\
& +\int_{\mathscr{B}}\left(6+2 \gamma_{f} \psi_{1}\left|\lambda_{m w}\right|\right)\|\mathbf{w}\|^{2} / 2+\int_{\mathscr{B}}\left(5+2 \gamma_{s} \phi_{1}\left|\lambda_{m u}\right|\right)\|\mathbf{u}\|^{2} / 2 \\
& +\int_{\mathscr{B}}\left(9 \varepsilon_{2}+\varepsilon_{3}-2\left(\mu_{1}+\kappa_{1}\right)\right)\|\widehat{\nabla} \mathbf{w}\|^{2} / 2+\int_{\mathscr{B}}\left(p_{f 1}-p_{f 2}\right) \psi_{2} \operatorname{div} \mathbf{w} \\
& +\int_{\mathscr{B}}\left(p_{f 1}-p_{f 2}\right)\left(\left(\nabla \psi_{2}\right) \cdot \mathbf{w}+\left(\nabla \phi_{2}\right) \cdot \mathbf{u}\right) \\
& +\int_{\mathscr{B}}\left[\left(p_{s 1}-p_{s 2}\right) \phi_{2}+\left(p_{s 1}-p_{f 1}\right) \phi\right] \operatorname{div} \mathbf{u} .
\end{aligned}
$$

See Remark 4.1 in regard to the bounds on $\|\mathbf{w}\|^{2}$ and $\|\mathbf{u}\|^{2}$. Note also that $v_{2}=w_{2}$ in $c_{8}$ (see Sec. 3). Let us turn our attention to the last three integrals. Using (4.2) and Graffi's argument (see the previous section) the last integral is bounded by

$$
\int_{\mathscr{B}}\left[c_{15} \phi^{2}+\|\mathbf{u}\|^{2}\right] / 2-\frac{d}{d t} \int_{\mathscr{B}} \frac{\beta_{3}}{\phi_{2}} \frac{\phi^{2}}{2},
$$

where $\beta_{3}=\left(p_{s}^{\prime}\left(\xi_{7}\right) \phi_{2}+p_{s 1}-p_{f 1}\right)$ and $c_{15}=\left(\left\|\beta_{3}\left(\nabla \phi_{2}\right) / \phi_{2}\right\|^{2}+\phi_{1}\left[\beta_{3} / \phi_{1} \phi_{2}\right]_{u_{1}}^{\circ}-\right.$ $\left.2 \beta_{3}\left(\operatorname{div} \mathbf{u}_{1}\right) / \phi_{2}\right)$. (Notice that now $p_{f}=p_{f}(\phi, \psi)$.)

Applying the Mean Value Theorem to $p_{f}$ we see that

$$
\begin{aligned}
p_{f 1}-p_{f 2} & :=p_{f}\left(\phi_{1}, \psi_{1}\right)-p_{f}\left(\phi_{2}, \psi_{2}\right)=\left(p_{f}\right)_{\phi}\left(\xi_{9}, \xi_{10}\right) \phi+\left(p_{f}\right)_{\psi}\left(\xi_{9}, \xi_{10}\right) \psi \\
& :=\left(p_{f}\right)_{\phi} \phi+\left(p_{f}\right)_{\psi} \psi,
\end{aligned}
$$

where $\left(p_{f}\right)_{\alpha}\left(\xi_{9}, \xi_{10}\right)=\left.\left(\partial p_{f} / \partial \alpha\right)\right|_{(\phi, \psi)=\left(\xi_{9}, \xi_{10}\right)}, \alpha=\phi$ or $\psi$, respectively, and $\xi_{9}, \xi_{10}$ $\in(0,1)$. Recall that $(\operatorname{div} \mathbf{w})^{2} \leq 9\|\hat{\nabla} \mathbf{w}\|^{2}$, hence integrals six and seven are bounded by

$$
\int_{\mathscr{B}}\left[c_{16} \phi^{2}+c_{17} \psi^{2}+2\|\mathbf{w}\|^{2}+2\|\mathbf{u}\|^{2}+9\left(\varepsilon_{6}+\varepsilon_{7}\right)\|\widehat{\nabla} \mathbf{w}\|^{2}\right] / 2,
$$

where $c_{16}=\left(2\left\|\left(p_{f}\right)_{\phi} \nabla \psi_{2}\right\|^{2}+\left(\left(p_{f}\right)_{\phi} \psi_{2}\right)^{2} / \varepsilon_{6}\right)$ and $c_{17}=\left(2\left\|\left(p_{f}\right)_{\psi} \nabla \psi_{2}\right\|^{2}+\right.$ $\left.\left(\left(p_{f}\right)_{\psi} \psi_{2}\right)^{2} / \varepsilon_{7}\right)$. Using (5.2) and (5.4) we see that (5.1) can be rewritten as

$$
\begin{aligned}
\frac{d}{d t} \int_{\mathscr{B}}\left[\gamma_{s} \phi_{1}\|\mathbf{u}\|^{2}+\gamma_{f} \psi_{1}\|\mathbf{w}\|^{2}+\beta_{3} \phi^{2} / \phi_{2}+\psi_{1} \psi^{2}\right] \\
\leq \int_{\mathscr{B}}\left[K_{5} \gamma_{s} \phi_{1}\|\mathbf{u}\|^{2}+K_{6} \gamma_{f} \psi_{1}\|\mathbf{w}\|^{2}+K_{7} \beta_{3} \phi^{2} / \phi_{2}+K_{8} \psi_{1} \psi^{2}\right] \\
\quad+\int_{\mathscr{B}}\left(9\left(\varepsilon_{2}+\varepsilon_{6}+\varepsilon_{7}\right)+\varepsilon_{3}-2\left(\mu_{1}+\kappa_{1}\right)\right)\|\hat{\nabla} \mathbf{w}\|^{2} / 2,
\end{aligned}
$$


where

$$
\begin{aligned}
& K_{5}=\sup _{\mathbf{x} \in \mathscr{B}}\left\{\left(8+2 \gamma_{s} \phi_{1}\left|\lambda_{m u}\right|\right) / \gamma_{s} \phi_{1}\right\}, \\
& K_{6}=\sup _{\mathbf{x} \in \mathscr{B}}\left\{\left(8+2 \gamma_{f} \psi_{1}\left|\lambda_{m w}\right|\right) / \gamma_{f} \psi_{1}\right\}, \\
& K_{7}=\sup _{\mathbf{x} \in \mathscr{B}}\left\{\left(2 c_{4}+c_{10}+c_{15}+c_{16}\right) \phi_{2} / \beta_{3}\right\}, \\
& K_{8}=\sup _{\mathbf{x} \in \mathscr{B}}\left\{\left(c_{2}+c_{3}+2 c_{5}+c_{8}+c_{17}\right) / \psi_{1}\right\} .
\end{aligned}
$$

Choose positive $\varepsilon_{2}, \varepsilon_{3}, \varepsilon_{6}$, and $\varepsilon_{7}$ such that $9\left(\varepsilon_{2}+\varepsilon_{6}+\varepsilon_{7}\right)+\varepsilon_{3}-2\left(\mu_{1}+\kappa_{1}\right)<0$. Setting $E_{2}(t)=\int_{\mathscr{B}}\left[\gamma_{s} \phi_{1}\|\mathbf{u}\|^{2}+\gamma_{f} \psi_{1}\|\mathbf{w}\|^{2}+\beta_{3} \phi^{2} / \phi_{2}+\psi_{1} \psi^{2}\right]$ and

$$
M_{2}=\sup _{t \in[0, T]}\left\{K_{5}, K_{6}, K_{7}, K_{8}\right\}
$$

we have that $d E_{2} / d t \leq M_{2} E_{2}$ and the desired result is obtained.

6. A limited viscosity fluid flow. Finally we will consider the problem of uniqueness for a limited viscosity fluid. We will assume that $p_{s}=p_{s}(\phi, \psi), p_{f}=p_{f}(\phi, \psi)$, and $\mathbf{u}=\mathbf{v}+\mathbf{w}$, and that $\mathscr{G}>0, \mu \geq 0$, and $\kappa>0$ satisfying the following relationship:

$$
\begin{gathered}
-\mathscr{G}_{1}^{2}+2\left(\kappa_{1}+\gamma_{s} \phi_{1} \mu_{1}+3 \gamma_{s} \phi_{1} \kappa_{1}+\gamma_{f} \psi_{1} \kappa_{1}\right) \mathscr{G}_{1}-\kappa_{1}^{2}-2 \kappa_{1}^{2} \gamma_{f} \psi_{1} \\
-\left(\gamma_{s} \phi_{1}\left(\mu_{1}+\kappa_{1}\right)-\gamma_{f} \psi_{1} \kappa_{1}\right)^{2}+2 \gamma_{s} \phi_{1} \kappa_{1}\left(\mu_{1}+\kappa_{1}\right)>0 .
\end{gathered}
$$

(See Sec. 5 for $\mathscr{G}=0$.) Then we have the following

TheOREM 3. Suppose that $\mathscr{G}>0, \mu \geq 0$, and $\kappa>0$ satisfy (C1). Then for every $T>0$ there is at most one classical solution of

$$
\begin{aligned}
& \gamma_{s} \phi \mathbf{u}_{t}+\gamma_{s} \phi \nabla \mathbf{u u}=-\nabla\left(p_{s} \phi\right)+\gamma_{s} \phi \mathbf{g}+\mathscr{F}(\mathbf{w}-\mathbf{u})-\mathscr{G} \operatorname{div}(\widehat{\nabla} \mathbf{w})+p_{f} \nabla \phi, \\
& \gamma_{f} \psi \mathbf{w}_{t}+\gamma_{f} \psi \nabla \mathbf{w} \mathbf{w}=-\nabla\left(p_{f} \psi\right)+\operatorname{div}(\mu \widehat{\nabla} \mathbf{w})+\gamma_{f} \psi \mathbf{g}-\mathscr{F}(\mathbf{w}-\mathbf{u}) \\
&+\mathscr{G} \operatorname{div}(\widehat{\nabla} \mathbf{w})-p_{f} \nabla \phi+\operatorname{div}(\kappa \widehat{\nabla} \mathbf{v}), \\
& \phi_{t}+\operatorname{div}(\phi \mathbf{u})=0, \quad \psi_{t}+\operatorname{div}(\psi \mathbf{w})=0,
\end{aligned}
$$

satisfying (3.1) and (3.2) on $[0, T]$.

Proof. The relationship (C1) will not be needed until the end of the proof. Eventually we will need Eqs. (3.7), (3.8), (3.18), and (3.19). We will also need one additional equation. To arrive at this additional equation, we begin by adding (3.3) and $(3.3)_{2}$. Taking the inner product of the result with $\left(\gamma_{s} \phi_{1} \mathbf{u}+\gamma_{f} \psi_{1} \mathbf{w}\right)$, integrating 
over $\mathscr{B}$, and applying the divergence theorem to the appropriate terms we see that

$$
\begin{aligned}
\frac{d}{d t} \int_{\mathscr{B}} & \left\|\gamma_{s} \phi_{1} \mathbf{u}+\gamma_{f} \psi_{1} \mathbf{w}\right\|^{2} / 2 \\
= & \int_{\mathscr{B}} \phi \phi_{1} \gamma_{s} \mathbf{b}_{2} \cdot \mathbf{u}+\int_{\mathscr{B}} \psi \psi_{1} \gamma_{f} \mathbf{a}_{2} \cdot \mathbf{w}+\int_{\mathscr{B}} \phi \psi_{1} \gamma_{f} \mathbf{b}_{2} \cdot \mathbf{w} \\
& +\int_{\mathscr{B}} \psi \phi_{1} \gamma_{s} \mathbf{a}_{2} \cdot \mathbf{u}-\int_{\mathscr{B}} \gamma_{s} \gamma_{f}\left(\psi_{1} \operatorname{div}\left(\phi_{1} \mathbf{u}_{1}\right)-\psi_{1} w_{1} \cdot \nabla \phi_{1}\right) \mathbf{u} \cdot \mathbf{w} \\
& -\int_{\mathscr{B}}\left[\gamma_{s} \gamma_{f} \phi_{1} \psi_{1}\left(\nabla \mathbf{u}_{2}+\left(\nabla \mathbf{w}_{2}\right)^{T}\right) \mathbf{u}\right] \cdot \mathbf{w}-\int_{\mathscr{B}} \gamma_{s}^{2} \phi_{1}^{2} \nabla \mathbf{u}_{2} \mathbf{u} \cdot \mathbf{u} \\
& -\int_{\mathscr{B}}\left[\gamma_{f}^{2} \psi_{1}^{2} \nabla \mathbf{w}_{2} \mathbf{w}\right] \cdot \mathbf{w}-\int_{\mathscr{B}} \gamma_{s}^{2} \phi_{1}^{2}\left(\operatorname{div} \mathbf{u}_{1}\right)\|\mathbf{u}\|^{2} / 2-\int_{\mathscr{B}} \gamma_{f}^{2} \psi^{2}\left(\operatorname{div} \mathbf{w}_{1}\right)\|\mathbf{w}\|^{2} / 2 \\
& -\int_{\mathscr{B}} \gamma_{s} \gamma_{f} \phi_{1} \psi_{1} \nabla \mathbf{u}\left(\mathbf{u}_{1}-\mathbf{w}_{1}\right) \cdot \mathbf{w}+\int_{\mathscr{B}}\left(\left(p_{s 1}-p_{s 2}\right) \phi_{2}+p_{s 1} \phi\right. \\
& \left.\quad+\left(p_{f 1}-p_{f 2}\right) \psi_{2}+p_{f 1} \psi\right) \operatorname{div}\left(\gamma_{s} \phi_{1} \mathbf{u}+\gamma_{f} \psi_{1} \mathbf{w}\right) \\
& -\int_{\mathscr{B}}\left[\left(\mu_{1}-\mu_{2}\right) \hat{\nabla} \mathbf{w}_{2}+\mu_{1} \hat{\nabla} \mathbf{w}+\left(\kappa_{1}-\kappa_{2}\right) \hat{\nabla} \mathbf{v}_{2}+\kappa_{1} \hat{\nabla} \mathbf{v}\right] \cdot \nabla\left(\gamma_{s} \phi_{1} \mathbf{u}+\gamma_{f} \psi_{1} \mathbf{w}\right),
\end{aligned}
$$

where $\mathbf{b}_{2}=\gamma_{s}\left(\mathbf{g}-\left(\mathbf{u}_{2}\right)_{t}-\nabla \mathbf{u}_{2} \mathbf{u}_{2}\right)$ and $\mathbf{a}_{2}=\gamma_{f}\left(\mathbf{g}-\left(\mathbf{w}_{2}\right)_{t}-\nabla \mathbf{w}_{2} \mathbf{w}_{2}\right)$. Applying the arithmetic-geometric mean inequality, (3.6), to the first six integrals on the right-hand side we see that the first ten integrals are bounded by

$$
\int_{\mathscr{B}}\left(c_{18} \phi^{2}+c_{19} \psi^{2}+c_{20}\|\mathbf{u}\|^{2}+c_{21}\|\mathbf{w}\|^{2}\right) / 2
$$

where $c_{18}=\left(\left(\phi_{1} \gamma_{s}\right)^{2}+\left(\psi_{1} \gamma_{f}\right)^{2}\right)\left\|\mathbf{b}_{2}\right\|^{2}, c_{19}=\left(\left(\phi_{1} \gamma_{s}\right)^{2}+\left(\psi_{1} \gamma_{f}\right)^{2}\right)\left\|\mathbf{a}_{2}\right\|^{2}, c_{20}=[2+$ $\left.\left(\gamma_{s} \gamma_{f}\right)^{2}\left(\psi_{1}\left\|\operatorname{div}\left(\phi_{1} \mathbf{u}_{1}\right)-\mathbf{w}_{1} \cdot \nabla \phi_{1}\right\|^{2}+\left(\psi_{1}\right)^{2}\left\|\nabla \mathbf{u}_{2}+\left(\nabla \mathbf{w}_{2}\right)^{T}\right\|^{2}\right)+\gamma_{s}^{2} \phi_{1}^{2}\left(2\left|\lambda_{m u}\right|-\operatorname{div} \mathbf{u}_{1}\right)\right]$, and $c_{21}=\left(4+\gamma_{f}^{2} \psi_{1}^{2}\left(2\left|\lambda_{m w}\right|-\operatorname{div} \mathbf{w}_{1}\right)\right)$.

Letting $c_{22}=\left\|\gamma_{s} \gamma_{f} \phi_{1} \psi_{1}\left(\mathbf{u}_{1}-\mathbf{w}_{1}\right)\right\|^{2}$, integral eleven can be bounded by

$$
\begin{aligned}
& -\int_{\mathscr{B}} \gamma_{s} \gamma_{f} \phi_{1} \psi_{1} \nabla \mathbf{u}\left(\mathbf{u}_{1}-\mathbf{w}_{1}\right) \cdot \mathbf{w} \\
& \quad \leq \int_{\mathscr{B}}\left[c_{22} \varepsilon_{8}\|\nabla \mathbf{u}\|^{2}+\|\mathbf{w}\|^{2} / \varepsilon_{8}\right] / 2 \\
& \quad \leq \int_{\mathscr{B}}\left[2 c_{22} \varepsilon_{8}\|\hat{\nabla} \mathbf{u}\|^{2}+\|\mathbf{w}\|^{2} / \varepsilon_{8}\right] / 2,
\end{aligned}
$$

by Korn's inequality (recall that $\mathbf{u}=0$ on $\partial \mathscr{B}$ ) (cf., e.g., Gurtin [13]).

Applying the Mean Value Theorem to $p_{s}$ we see that

$$
\begin{aligned}
p_{s 1}-p_{s 2} & :=p_{s}\left(\phi_{1}, \psi_{1}\right)-p_{s}\left(\phi_{2}, \psi_{2}\right)=\left(p_{s}\right)_{\phi}\left(\xi_{11}, \xi_{12}\right) \phi+\left(p_{s}\right)_{\psi}\left(\xi_{11}, \xi_{12}\right) \psi \\
& :=\left(p_{s}\right)_{\phi} \phi+\left(p_{s}\right)_{\psi} \psi
\end{aligned}
$$

where $\xi_{11} \xi_{12} \in(0,1)$. Using (5.3) and the above we have that integral twelve 
becomes

$$
\begin{aligned}
& \int_{\mathscr{B}}\left(c_{23} \phi+c_{24} \psi\right)\left(\gamma_{s} \mathbf{u} \cdot \nabla \phi_{1}+\gamma_{f} \mathbf{w} \cdot \nabla \psi_{1}+\gamma_{s} \phi_{1} \operatorname{div} \mathbf{u}+\gamma_{f} \psi_{1} \operatorname{div} \mathbf{w}\right) \\
& \leq \int_{\mathscr{B}}\left[c_{25} \phi^{2}+c_{26} \psi^{2}+2\|\mathbf{u}\|^{2}+2\|\mathbf{w}\|^{2}+9\left(\varepsilon_{9}+\varepsilon_{11}\right)\|\widehat{\nabla} \mathbf{u}\|^{2}+9\left(\varepsilon_{10}+\varepsilon_{12}\right)\|\hat{\nabla} \mathbf{w}\|^{2}\right] / 2,
\end{aligned}
$$

where $c_{23}=\left(\left(p_{s}\right)_{\phi} \phi_{2}+p_{s 1}+\left(p_{f}\right)_{\phi} \psi_{2}\right), c_{24}=\left(\left(p_{s}\right)_{\psi} \phi_{2}+\left(p_{f}\right)_{\psi} \psi_{2}+p_{f 1}\right), c_{25}=$ $c_{23}^{2}\left(\left\|\gamma_{s} \nabla \phi_{1}\right\|^{2}+\left\|\gamma_{f} \nabla \psi_{1}\right\|^{2}+\left(\phi_{1} \gamma_{s}\right)^{2} / \varepsilon_{9}+\left(\gamma_{f} \psi_{1}\right)^{2} / \varepsilon_{10}\right)$, and

$$
c_{26}=c_{24}^{2}\left(\left\|\gamma_{s} \nabla \phi_{1}\right\|^{2}+\left\|\gamma_{f} \nabla \psi_{1}\right\|^{2}+\left(\gamma_{s} \phi_{1}\right)^{2} / \varepsilon_{11}+\left(\gamma_{f} \psi_{1}\right)^{2} / \varepsilon_{12}\right)
$$

Let us now turn our attention to the last integral in (6.1). Using $(3.13)_{3,4}$ this integral can be rewritten as

$$
\begin{aligned}
-\int_{\mathscr{B}}[ & \gamma_{s}(\psi A+B) \cdot \mathbf{u} \otimes \nabla \phi_{1}+\gamma_{s} \phi_{1}(\psi A+B) \cdot \hat{\nabla} \mathbf{u} \\
& \left.+\gamma_{f}(\psi A+B) \cdot \mathbf{w} \otimes \nabla \psi_{1}+\gamma_{f} \psi_{1}(\psi A+B) \cdot \hat{\nabla} \mathbf{w}\right],
\end{aligned}
$$

where $A=\left(\mu^{\prime}\left(\xi_{5}\right) \hat{\nabla} \mathbf{w}_{2}+\kappa^{\prime}\left(\xi_{6}\right) \widehat{\nabla} \mathbf{v}_{2}\right)$ and $B=\left(\mu_{1} \widehat{\nabla} \mathbf{w}+\kappa_{1} \widehat{\nabla} \mathbf{v}\right)$. Now

$$
-\int_{\mathscr{B}}\left[\gamma_{s} \psi A \cdot \mathbf{u} \otimes \nabla \phi_{1}+\gamma_{f} \psi A \cdot \mathbf{w} \otimes \nabla \psi_{1}\right] \leq \int\left[c_{27} \psi^{2}+\|\mathbf{u}\|^{2}+\|\mathbf{w}\|^{2}\right] / 2,
$$

where $c_{27}=\left\|\gamma_{s} A \nabla \phi_{1}\right\|^{2}+\left\|\gamma_{f} A \nabla \psi_{1}\right\|^{2}$. Also

$$
\begin{gathered}
-\int_{\mathscr{B}}\left[\gamma_{s} B \cdot \mathbf{u} \otimes \nabla \phi_{1}+\gamma_{s} \phi_{1} \psi A \cdot \widehat{\nabla} \mathbf{u}+\gamma_{f} B \cdot \mathbf{w} \otimes \nabla \psi_{1}+\gamma_{f} \psi_{1} \psi A \cdot \widehat{\nabla} \mathbf{w}\right] \\
\leq \int_{\mathscr{B}}\left[c_{28} \psi^{2}+c_{29}\|\mathbf{u}\|^{2}+c_{30}\|\mathbf{w}\|^{2}\right. \\
\left.\quad+\left(\varepsilon_{13}+\varepsilon_{17}+\varepsilon_{18}\right)\|\widehat{\nabla} \mathbf{u}\|^{2}+\left(\varepsilon_{14}+\varepsilon_{15}+\varepsilon_{16}\right)\|\widehat{\nabla} \mathbf{w}\|^{2}\right] / 2,
\end{gathered}
$$

where $c_{28}=\left(\left\|\gamma_{s} \phi_{1} A\right\|^{2} / \varepsilon_{13}+\left\|\gamma_{f} \psi_{1} A\right\|^{2} / \varepsilon_{14}\right), \quad c_{29}=\left(\left\|\gamma_{s}\left(\mu_{1}+\kappa_{1}\right) \nabla \phi_{1}\right\|^{2} / \varepsilon_{15}+\right.$ $\left.\left\|\gamma_{s} \kappa_{1} \nabla \phi_{1}\right\|^{2} / \varepsilon_{17}\right)$, and $c_{30}=\left(\left\|\gamma_{f}\left(\mu_{1}+\kappa_{1}\right) \nabla \psi_{1}\right\|^{2} / \varepsilon_{16}+\left\|\gamma_{f} \kappa_{1} \nabla \psi_{1}\right\|^{2} / \varepsilon_{18}\right)$. Then last terms to be considered are

$$
\begin{aligned}
-\int_{\mathscr{B}}\left[\gamma_{s} \phi_{1} B \cdot \nabla \mathbf{u}+\gamma_{f} \psi_{1} B \cdot \nabla \mathbf{w}\right] \\
=-\int_{\mathscr{B}}\left[\gamma_{s} \phi_{1}\left(\mu_{1}+\kappa_{1}\right) \hat{\nabla} \mathbf{w} \cdot \hat{\nabla} \mathbf{u}+\gamma_{s} \phi_{1} \kappa_{1}\|\widehat{\nabla} \mathbf{u}\|^{2}\right. \\
\left.\quad+\gamma_{f} \psi_{1}\left(\mu_{1}+\kappa_{1}\right)\|\widehat{\nabla} \mathbf{w}\|^{2}+\gamma_{f} \psi_{1} \kappa_{1} \widehat{\nabla} \mathbf{u} \cdot \widehat{\nabla} \mathbf{w}\right]
\end{aligned}
$$


Thus, using (6.2)-(6.7) we see that

$$
\begin{aligned}
\frac{d}{d t} \int_{\mathscr{B}} & \left\|\gamma_{s} \phi_{1} \mathbf{u}+\gamma_{f} \psi_{1} \mathbf{w}\right\|^{2} \\
\leq & \int_{\mathscr{B}}\left[\left(c_{18}+c_{25}\right) \phi^{2}+\left(c_{19}+c_{26}+c_{27}+c_{28}\right) \psi^{2}\right. \\
& \left.\quad+\left(c_{20}+3+c_{29}\right)\|\mathbf{u}\|^{2}+\left(3+c_{21}+c_{30}+1 / \varepsilon_{8}\right)\|\mathbf{w}\|^{2}\right] \\
& +\int_{\mathscr{B}}\left[\left(\delta_{1}-2 \gamma_{s} \phi_{1} \kappa_{1}\right)\|\hat{\nabla} \mathbf{u}\|^{2}+\left(\delta_{2}-2 \gamma_{f} \psi_{1}\left(\mu_{1}+\kappa_{1}\right)\right)\|\hat{\nabla} \mathbf{w}\|^{2}\right] \\
& +2 \int_{\mathscr{B}}\left[-\gamma_{s} \phi_{1}\left(\mu_{1}+\kappa_{1}\right)-\gamma_{f} \psi_{1} \kappa_{1}\right] \hat{\nabla} \mathbf{u} \cdot \hat{\nabla} \mathbf{w},
\end{aligned}
$$

where $\delta_{1}=\left(2 c_{22} \varepsilon_{8}+9\left(\varepsilon_{9}+\varepsilon_{11}\right)+\varepsilon_{13}+\varepsilon_{17}+\varepsilon_{18}\right)$ and $\delta_{2}=\left(9\left(\varepsilon_{10}+\varepsilon_{12}\right)+\varepsilon_{14}+\varepsilon_{15}+\varepsilon_{16}\right)$. Adding Eqs. (3.7), (3.8), (3.18), and (3.19) and applying (3.6) to the appropriate integral we arrive at

$$
\begin{aligned}
& \frac{d}{d t} \int_{\mathscr{B}}\left(\phi_{1} \phi^{2}+\psi_{1} \psi^{2}+\gamma_{s} \phi_{1}\|\mathbf{u}\|^{2}+\gamma_{f} \psi_{1}\|\mathbf{w}\|^{2}\right) \\
& \leq \int_{\mathscr{B}}\left[\left(c_{1}+2 c_{4}+2 c_{6}+c_{10}+c_{16}+c_{31}\right) \phi^{2}\right. \\
& \quad+\left(c_{2}+c_{3}+2 c_{5}+2 c_{7}+c_{8}+c_{17}+c_{32}\right) \psi^{2} \\
& \left.\quad+\left(8+2 \gamma_{s} \phi_{1}\left|\lambda_{m u}\right|+c_{11}\right)\|\mathbf{u}\|^{2}+\left(8+2 \gamma_{f} \psi_{1}\left|\lambda_{m w}\right|+c_{9}\right)\|\mathbf{w}\|^{2}\right] \\
& \quad+\int_{\mathscr{B}}\left[\left(9 \varepsilon_{1}+9 \varepsilon_{19}+9 \varepsilon_{20}\right)\|\hat{\nabla} \mathbf{u}\|^{2}\right. \\
& \left.\quad+\left(9 \varepsilon_{2}+\varepsilon_{3}+\varepsilon_{4}+\varepsilon_{5}+9 \varepsilon_{6}+9 \varepsilon_{7}-2\left(\mathscr{G}_{1}+\mu_{1}+\kappa_{1}\right)\right)\|\hat{\nabla} \mathbf{w}\|^{2}\right] \\
& \quad+\int_{\mathscr{B}} 2\left(\mathscr{G}_{1}-\kappa_{1}\right) \hat{\nabla} \mathbf{u} \cdot \hat{\nabla} \mathbf{w},
\end{aligned}
$$

where $c_{31}=\left(\left(p_{s}\right)_{\phi} \phi_{2}+\left(p_{s 1}-p_{f 1}\right)\right)^{2} / \varepsilon_{19}, \quad c_{32}=\left(\left(p_{s}\right)_{\psi} \phi_{2}\right)^{2} / \varepsilon_{20}$, and we have used (5.4). Adding this to (6.8) we have

$$
\begin{aligned}
\frac{d}{d t} \int_{\mathscr{B}}\left[\gamma_{s} \phi_{1}\|\mathbf{u}\|^{2}+\gamma_{f} \psi_{1}\|\mathbf{w}\|^{2}+\left\|\gamma_{s} \phi_{1} \mathbf{u}+\gamma_{f} \psi_{1} \mathbf{w}\right\|^{2}+\phi_{1} \phi^{2}+\psi_{1} \psi^{2}\right] \\
\leq \int_{\mathscr{B}}\left[K_{9} \gamma_{s} \phi_{1}\|\mathbf{u}\|^{2}+K_{10} \gamma_{f} \psi_{1}\|\mathbf{w}\|^{2}+\left\|\gamma_{s} \phi_{1} \mathbf{u}+\gamma_{f} \psi_{1} \mathbf{w}\right\|^{2}\right. \\
\left.+K_{11} \phi_{1} \phi^{2}+K_{12} \psi_{1} \psi^{2}\right] \\
+\int_{\mathscr{B}}\left[\hat{\delta}_{1}-2 \gamma_{s} \phi_{1} \kappa_{1}\right)\|\hat{\nabla} \mathbf{u}\|^{2} \\
+2\left(-\gamma_{s} \phi_{1}\left(\mu_{1}+\kappa_{1}\right)-\gamma_{f} \psi_{1} \kappa_{1}+\mathscr{G}_{1}-\kappa_{1}\right) \hat{\nabla} \mathbf{u} \cdot \hat{\nabla} \mathbf{w} \\
\left.+\left(\hat{\delta}_{2}-2 \gamma_{f} \psi_{1}\left(\mu_{1}+\kappa_{1}\right)-2\left(\mathscr{G}_{1}+\mu_{1}+\kappa_{1}\right)\right)\|\hat{\nabla} \mathbf{w}\|^{2}\right],
\end{aligned}
$$


where $\hat{\delta}_{1}=\left(\delta_{1}+9 \varepsilon_{1}+9 \varepsilon_{19}+9 \varepsilon_{20}\right), \hat{\delta}_{2}=\left(\delta_{2}+9 \varepsilon_{2}+\varepsilon_{3}+\varepsilon_{4}+\varepsilon_{5}+9 \varepsilon_{6}+9 \varepsilon_{7}\right)$, and

$$
\begin{aligned}
K_{9} & =\sup _{\mathbf{x} \in \mathscr{B}}\left\{\left(11+2 \gamma_{s} \phi_{1}\left|\lambda_{m u}\right|+c_{11}+c_{20}+c_{29}\right) / \gamma_{s} \phi_{1}\right\}, \\
K_{10} & =\sup _{\mathbf{x} \in \mathscr{B}}\left\{\left(11+2 \gamma_{f} \psi_{1}\left|\lambda_{m w}\right|+c_{9}+c_{21}+c_{30}+1 / \varepsilon_{8}\right) / \gamma_{f} \psi_{1}\right\}, \\
K_{11} & =\sup _{\mathbf{x} \in \mathscr{B}}\left\{\left(c_{1}+2 c_{4}+2 c_{6}+c_{10}+c_{16}+c_{18}+c_{25}+c_{31}\right) / \phi_{1}\right\}, \\
K_{12} & =\sup _{\mathbf{x} \in \mathscr{B}}\left\{\left(c_{2}+c_{3}+2 c_{5}+2 c_{7}+c_{8}+c_{17}+c_{19}+c_{26}+c_{27}+c_{28}+c_{32}\right) / \psi_{1}\right\} .
\end{aligned}
$$

Remark 6.1. Notice that $\hat{\delta}_{1}$ and $\hat{\delta}_{2}$ depend upon $\varepsilon_{i}, i=1, \ldots, 20$, and that each $\varepsilon_{i}>0$ can be made arbitrarily small. Since we are also dividing by these quantities in the various $\phi^{2}, \psi^{2},\|\mathbf{u}\|^{2}$, and $\|\mathbf{w}\|^{2}$ terms, we require that each $\varepsilon_{i}$ be nonzero.

Consider now the last integral in (6.9). If it is nonpositive then we are done. Let

$$
E_{3}(t)=\int_{\mathscr{B}}\left[\gamma_{s} \phi_{1}\|\mathbf{u}\|^{2}+\gamma_{f} \psi_{1}\|\mathbf{w}\|^{2}+\left\|\gamma_{s} \phi_{1} \mathbf{u}+\gamma_{f} \psi_{1} \mathbf{w}\right\|^{2}+\phi_{1} \phi^{2}+\psi_{1} \psi^{2}\right]
$$

and $M_{3}=\sup _{t \in[0, T]}\left\{1, K_{9}, K_{10}, K_{11}, K_{12}\right\}$. Then $d E_{3} / d t \leq M_{3} E_{3}$ and $E_{3}(t) \leq$ $E_{3}(0) \exp \left(M_{3} t\right) \equiv 0$. Thus $\phi(\mathbf{x}, t)=\psi(\mathbf{x}, t)=0, \mathbf{u}(\mathbf{x}, t)=\mathbf{w}(\mathbf{x}, t)=\mathbf{0}$, and whence $\phi_{1}(\mathbf{x}, t)=\phi_{2}(\mathbf{x}, t), \quad \psi_{1}(\mathbf{x}, t)=\psi_{2}(\mathbf{x}, t), \quad \mathbf{u}_{1}(\mathbf{x}, t)=\mathbf{u}_{2}(\mathbf{x}, t)$, and $\mathbf{w}_{1}(\mathbf{x}, t)=$ $\mathbf{w}_{2}(\mathbf{x}, t)$.

Thus all that is left to show is that for some choice of $\mathscr{G}, \mu$, and $\kappa$, the following integral (which is the second integral in (6.9)) is negative:

$$
\begin{array}{r}
\int_{\mathscr{B}}\left[\left(\hat{\delta}_{1}-2 \gamma_{s} \phi_{1} \kappa_{1}\right)\|\hat{\nabla} \mathbf{u}\|^{2}+2\left(\mathscr{G}_{1}-\kappa_{1}-\gamma_{f} \psi_{1} \kappa_{1}-\gamma_{s} \phi\left(\mu_{1}+\kappa\right)\right) \hat{\nabla} \mathbf{u} \cdot \hat{\nabla} \mathbf{w}\right. \\
\left.+\left(\hat{\delta}_{2}-2 \gamma_{f} \psi_{1}\left(\mu_{1}+\kappa_{1}\right)-2\left(\mathscr{G}_{1}+\mu_{1}+\kappa_{1}\right)\right)\|\hat{\nabla} \mathbf{w}\|^{2}\right] .
\end{array}
$$

Let $a=2 \gamma_{s} \phi_{1} \kappa_{1}, b=\left(-\mathscr{G}_{1}+\kappa_{1}+\gamma_{s} \phi_{1}\left(\mu_{1}+\kappa_{1}\right)+\gamma_{f} \psi_{1} \kappa_{1}\right)$, and

$$
c=2\left(\mathscr{G}_{1}+\mu_{1}+\kappa_{1}+\gamma_{f} \psi_{1}\left(\mu_{1}+\kappa_{1}\right)\right) \text {. }
$$

Then (6.10) becomes

$$
\int_{\mathscr{B}}\left[-a\|\hat{\nabla} \mathbf{u}\|^{2}-2 b \hat{\nabla} \mathbf{u} \cdot \hat{\nabla} \mathbf{w}-c\|\hat{\nabla} \mathbf{w}\|^{2}\right]+\int_{\mathscr{B}}\left[\hat{\delta}_{1}\|\hat{\nabla} \mathbf{u}\|^{2}+\hat{\delta}_{2}\|\hat{\nabla} \mathbf{w}\|^{2}\right] .
$$

If it can be shown that the first integral is strictly negative then we are done. Simply choose $\hat{\delta}_{1}$ and $\hat{\delta}_{2}$ such that (6.11) is nonpositive, i.e., if $b \hat{\nabla} \mathbf{u} \cdot \hat{\nabla} \mathbf{w}>0$ then choose $\hat{\delta}_{1}$ and $\hat{\delta}_{2}$ such that $-a+\hat{\delta}_{1}<0$ and $-c+\hat{\delta}_{2}<0$.

Showing the first integral is strictly negative is equivalent to showing

$$
\int_{\mathscr{B}} a\|\hat{\nabla} \mathbf{u}\|^{2}+2 b \hat{\nabla} \mathbf{u} \cdot \hat{\nabla} \mathbf{w}+c\|\hat{\nabla} \mathbf{w}\|^{2}
$$

is strictly positive. If $a, c>0$ and $b^{2}<a c$, then the above integral is positive. The first two conditions are obviously true. We need to determine conditions on $\mu, \kappa$, and $\mathscr{G}$ to ensure that $a c-b^{2}>0$, i.e.,

$$
\begin{gathered}
-\mathscr{G}_{1}^{2}+2\left(\kappa_{1}+\gamma_{s} \phi_{1} \mu_{1}+3 \gamma_{s} \phi_{1} \kappa_{1}+\gamma_{f} \psi_{1} \kappa_{1}\right) \mathscr{G}_{1}-\kappa_{1}^{2}-2 \kappa_{1}^{2} \gamma_{f} \psi_{1} \\
-\left(\gamma_{s} \phi_{1}\left(\mu_{1}+\kappa_{1}\right)-\gamma_{f} \psi_{1} \kappa_{1}\right)^{2}+2 \gamma_{s} \phi_{1} \kappa_{1}\left(\mu_{1}+\kappa_{1}\right)>0,
\end{gathered}
$$

which is condition $(\mathrm{C} 1)$. 
Thus for any $\kappa_{1}>0$ and any $\mu_{1} \geq 0$ we need to choose $\mathscr{G}_{1}>0$ such that the above inequality holds. To show that there exists $\mathscr{G}_{1}$ such that the above inequality holds we need to show that the quadratic equation (in $\mathscr{G}_{1}$ ) has two real roots with at least one positive root. This will be true if the discriminant is positive, i.e.,

$$
\begin{aligned}
4\left(\kappa_{1}\right. & \left.+\gamma_{s} \phi_{1} \mu_{1}+3 \gamma_{s} \phi_{1} \kappa_{1}+\gamma_{f} \psi_{1} \kappa_{1}\right)^{2} \\
& +4\left(2 \gamma_{s} \phi_{1} \kappa_{1}\left(\mu_{1}+\kappa_{1}\right)-\kappa_{1}^{2}-2 \gamma_{f} \psi_{1} \kappa_{1}^{2}-\left(\gamma_{s} \phi_{1}\left(\mu_{1}+\kappa_{1}\right)-\gamma_{f} \psi_{1} \kappa_{1}\right)^{2}\right)>0 .
\end{aligned}
$$

This in fact does hold. Furthermore, the maximum of this quadratic function occurs at

$$
\mathscr{G}_{1 m}=\kappa_{1}+\gamma_{s} \phi_{1} \mu_{1}+3 \gamma_{s} \phi_{1} \kappa_{1}+\gamma_{f} \psi_{1} \kappa_{1},
$$

which is positive. Thus, if $\mathscr{G}_{1}^{+}$and $\mathscr{G}_{1}^{-}$denote the larger and smaller root of this equation, we see that $\mathscr{G}_{1}^{+}>0$. (In order for $\mathscr{G}_{1}^{-}$to be positive we would need the constant term to be negative, i.e., the discriminant squared needs to be less than the coefficient of $\mathscr{G}_{1}$. This may or may not be true.) Let $\mathscr{G}_{1}^{0}=\max \left\{0, \mathscr{G}_{1}^{-}\right\}$. Then choose $\mathscr{G} \in\left(\mathscr{G}_{1}^{0}, \mathscr{G}_{1}^{+}\right)$and the desired result holds. Thus, there is at most one solution to the initial-boundary value problem.

REMARK 6.2. Given any $\mu$ and $\kappa$ we now have a range of values for $\mathscr{G}$ to take in order to ensure uniqueness, i.e., if $\mathscr{G} \in\left(\mathscr{G}_{1}^{0}, \mathscr{G}_{1}^{+}\right)$then uniqueness holds.

REMARK 6.3. Note that $\kappa$ must be positive for this method to work. Otherwise, if $\kappa=0$ and $\mathscr{G}$ and $\mu$ are nonzero then (6.11) is positive. (Here (C1) becomes $-\left(\mathscr{G}_{1}-\gamma_{s} \phi_{1} \mu_{1}\right)^{2}>0$, which cannot happen.) If $\kappa$ and $\mathscr{G}$ are both zero then the methods of Sec. 5 hold.

REMARK 6.4. Another way to look at this problem is: given any $\mathscr{G} \in(\alpha, \beta)$, where $\alpha, \beta>0$, we need to choose $\mu \geq 0$ and $\kappa>0$ such that $(\mathrm{C} 1)$ holds, i.e., given $\mathscr{G}$ it is necessary to limit the values that $\mu$ and $\kappa$ can take on.

7. Conclusions. There are still many open questions left on this problem. In particular, it would be interesting to determine what would happen if $\kappa \equiv 0$, and $\mathscr{G} \neq 0$ or the case when $\mathscr{G}$ does not depend on the values of $\mu$ and $\kappa$.

In this paper it was shown that if $\mathscr{G}, \mu$, and $\kappa$ satisfy a certain relationship (see Sec. 6) then uniqueness holds. What physical significance (if any) does this condition have?

In the original paper by McTigue, Givler, and Nunziato [1] they considered the velocity of the mixture to be

$$
\rho v=\gamma_{s} \phi u+\gamma_{f} \psi w
$$

where $\rho=\phi \gamma_{s}+\psi \gamma_{f}$. The above analysis still holds by letting $\hat{\mu}=\mu+\gamma_{f} \psi /\left(\gamma_{s} \phi+\right.$ $\left.\gamma_{f} \psi\right), \hat{\kappa}=\gamma_{s} \phi /\left(\gamma_{s} \phi+\gamma_{f} \psi\right)$, and recalling that $\phi+\psi=1$. They also considered

$$
\mathscr{F}=\alpha_{1} 1+\alpha_{2} \hat{\nabla} \mathbf{w}, \quad \mathscr{G}=\beta_{1} 1+\beta_{2} \hat{\nabla} w,
$$

where $\alpha_{i}, \beta_{i}$ are functions of $\phi$ and $\psi$. Here $\alpha_{1}$ is associated with the Stokes drag force and $\alpha_{2}$ with the lift due to interaction of the slip velocity with the mean shearing analyzed by Saffman. The coefficient $\beta_{1}$ is associated with the Faxén force. Here the problem is more difficult and still needs to be analyzed. 
Finally we have assumed a special form of the pressure, i.e., $p_{s}=p_{s}(\phi, \psi)$ and $p_{f}=p_{f}(\phi, \psi)$. McTigue, Givler, and Nunziato consider a more general constitutive equation of the form

$$
p_{s}=p_{f}+\tau+\omega\|\mathbf{u}-\mathbf{w}\|^{2}+\zeta \operatorname{tr}(\widehat{\nabla} \mathbf{w}),
$$

where the Brownian pressure $\tau$ is a function of the particle concentration, and $\omega$ and $\zeta$ are taken to be constants. The coefficient $\zeta$ may be viewed as a bulk viscosity.

In the last two cases it would be interesting to determine if the solution is unique. Notice that an even more interesting question that still needs to be answered is to determine if the problem is well-posed and has a solution.

Acknowledgments. The formulation of this problem is due to J. Nunziato. I would also like to thank R. C. Givler and S. L. Passman for their insights into the problem and for our numerous discussions. This work was initiated while the author was a member of the Institute for Mathematics and Its Applications at the University of Minnesota and was partially supported by the National Science Foundation under grant number DMS-8810618.

\section{REFERENCES}

[1] D. F. McTigue, R. C. Givler, and J. W. Nunziato, Rheological effects of non-uniform particle distributions in dilute suspensions, J. Rheol. 5, 1053-1076 (1986)

[2] A. Einstein, Eine neue Bestimmung der Moleküldimensionen, Ann. Physik 19, 289-306 (1906).

[3] G. K. Batchelor, Sedimentation in a dilute dispersion of spheres, J. Fluid Mech. 52, 245-268 (1972)

[4] G. K. Batchelor and J. T. Green, The determination of the bulk stress in a suspension of spherical particles to order $c^{2}$, J. Fluid Mech. 56, 401-427 (1972)

[5] D. Bedeaux, The effective viscosity for a suspension of spheres, J. Col. Interface Sci. 118, 80-86 (1987)

[6] N. A. Frankel and A. Acrivos, On the viscosity of a concentrated suspension of solid spheres, Chem. Engrg. Sci. 22, 847-853 (1967)

[7] F. Parsi and F. Gadala-Maria, Fore-and-aft asymmetry in a concentrated suspension of solid spheres, J. Rheol. 8, 725-732 (1987)

[8] C. Segre and A. Silberberg, Behaviour of macroscopic rigid spheres in Poiseuille flow. Part 1. Determination of local concentration by statistical analysis of particle passages through crossed light beams J. Fluid Mech. 14, 115-135 (1962)

[9] A Karnis, H. L. Goldsmith, and S. G. Mason, The flow of suspensions through tubes V. Inertial effects, Canad. J. Chem. Engrg. 44, 181-193 (1966)

[10] H. Aoki, Y. Kurosaki, and H. Anzai, Study on the tubular pinch effect in a pipe flow I. Lateral migration of a single particle in laminar Poiseuille flow, Bull. JSME 22, 206-212 (1979)

[11] W. O. Williams On the theory of mixtures, Arch. Rational Mech. Anal. 51, 239-260 (1973)

[12] D. Graff, Il teorema di unicità nella dinamica dei fluidi compressible, JRMA 2, 99-106 (1973)

[13] M. E. Gurtin, The Linear Theory of Elasticity, Handbuch der Physik VI a/2 (C. Truesdell, ed.), Springer-Verlag, Berlin, 1972 